\title{
Outcomes of Endoscopic Submucosal Dissection for Subepithelial Lesions Localized Within the Submucosa, Including Neuroendocrine Tumors: A Multicenter Prospective Study
}

\author{
Hideki Kobara $^{1}$, Yoichi Miyaoka ${ }^{2}$, Yoshio Ikeda ${ }^{3}$, Takayoshi Yamada ${ }^{4}$, Masashi Takata ${ }^{4}$, Shintaro Fujihara ${ }^{1}$, Noriko \\ Nishiyama $^{1}$, Koji Fujita ${ }^{1}$, Joji Tani ${ }^{1}$, Nobuya Kobayashi ${ }^{1}$, Taiga Chiyo ${ }^{1}$, Tatsuo Yachida ${ }^{1}$, Keiichi Okano ${ }^{5}$, Yasuyuki \\ Suzuki $^{5}$, Hirohito Mori ${ }^{1}$, Tsutomu Masaki ${ }^{1}$
}

\author{
1) Department of \\ Gastroenterology and \\ Neurology, Faculty of \\ Medicine, Kagawa University, \\ Kita, Kagawa \\ 2) Division of Endoscopy, \\ Shimane Prefectural Central \\ Hospital, Izumo, Shimane \\ 3) Division of Endoscopy, \\ Ehime University, Matsuyama, \\ Ehime \\ 4) Department of \\ Gastroenterology, Kochi \\ Health Sciences Center, Kochi, \\ Japan \\ 5) Department of \\ Gastroenterological Surgery, \\ Faculty of Medicine, Kagawa \\ University, Kita, Kagawa, \\ Japan
}

\begin{abstract}
Background \& Aims: Endoscopic submucosal dissection (ESD) seems to be a reasonable option for gastrointestinal subepithelial lesions (SELs) localized within the submucosa. Indications for ESD include small neuroendocrine tumors (NETs) and indeterminate SELs. However, the prospective data regarding ESD and surveillance remain unclear. This study was performed to prospectively investigate the outcomes of ESD, including organ-specific outcomes and the mid-term prognosis.

Methods: This prospective multicenter study included 57 patients who underwent ESD for SELs localized within the submucosa [definite NETs $(n=42)$ and indeterminate SELs $(n=15)$ ]. The efficacy and safety of ESD were evaluated in the whole cohort and in subgroups (NETs and indeterminate SELs). All patients were followed up.

Results: The rates of en bloc resection, curative resection, and complications were $98.2 \%, 66.7 \%$, and $7.7 \%$ for the overall population ( $n=57) ; 100 \%, 61.9 \%$, and $2.4 \%$ for NETs $(n=42)$; and $93.3 \%, 80.0 \%$, and $20.0 \%$ for indeterminate SELs $(n=15)$, respectively. The rates of curative resection for NETs were poorer in the stomach $(20 \%, n=5)$ and duodenum $(33 \%, n=3)$ than in the rectum $(71 \%, n=34)$. Including 11 of 16 patients with NETs who underwent a conservative approach resulting in non-curative resection, no patients developed tumor recurrence during the follow-up period (median, 24.5 months; range, 1-60 months). ESD followed by surveillance demonstrated acceptable mid-term outcomes for non-curative NETs.

Conclusions: ESD can be an efficient therapy for SELs localized within the submucosa. However, gastric and duodenal ESD for NETs may be limited in terms of its curative and technical aspects. Clinicians should be aware of the potential complications of ESD for indeterminate SELs.
\end{abstract}

Key words: endoscopic submucosal dissection - gastrointestinal subepithelial lesions - small neuroendocrine tumors.

Abbreviations: EMR: endoscopic mucosal resection; ESD: endoscopic submucosal dissection; EUS: endosonography; GI: gastrointestinal; MP: muscularis propria; NET: neuroendocrine tumor; SEL: subepithelial lesion; SM: submucosa.

\section{INTRODUCTION}

Endoscopic submucosal dissection (ESD), which can facilitate dissection of the submucosa (SM) from the underlying muscularis propria (MP), enables en bloc resection and has become the standard procedure for resecting early gastrointestinal (GI) neoplasms [1-3]. In principle, ESD can be applied for complete resection of subepithelial lesions (SELs) located within the SM. Indications for ESD include small GI neuroendocrine tumors (NETs), indeterminate SELs for which malignant potential cannot be ruled out [e.g., granular cell tumor and mucosa-association lymphoid tissue (MALT) lymphoma], and symptomatic SELs. Gastrointestinal NETs occur in approximately 1.95 to 2.50 people per 100,000 population [4]. However, the United States Surveillance Epidemiology and End Results program recently suggested that the incidence and prevalence of NETs have substantially increased during the past three decades. This may partly reflect the increased number of diagnoses of benign and incidentally identified lesions because of the increased availability of advanced endoscopic and radiological imaging [5]. Smallersized $(\leq 10-\mathrm{mm})$ NETs with an extremely low risk of metastasis 
[6] are treated endoscopically. Endoscopic resection is indicated for NET grade 1 (NET G1) that is $\leq 10 \mathrm{~mm}$ in size, without MP layer invasion (T1), and without lymphatic or venous invasion. However, special techniques are required to achieve curative resection with negative vertical margins [7] because most of these tumors extend into the SM layer [8]. Thus, ESD is recommended because it enables secure submucosal dissection directly above the MP and precise histological assessment of the resected specimen, which are advantages over conventional endoscopic mucosal resection (EMR) $[9,10]$. Moreover, ESD can provide an accurate diagnosis as well as curative resection of indeterminate SELs $[11,12]$, and symptomatic SELs [13, 14]. However, prospective data regarding the ESD procedure and surveillance are not available. Additionally, no consensus regarding gastric and duodenal ESD as NETs treatment strategy has been established, although rectal ESD is recommended according to sufficient evidence.

We prospectively investigate the technical and clinical outcomes of ESD for SELs located within the SM, including organ-specific outcomes and the mid- to long-term prognosis.

\section{METHODS}

\section{Study design}

This prospective observational study was conducted at four medical centers in the Shikoku areas and in Shimane of Japan. The study involved 57 patients who underwent ESD for SELs localized within the SM, including definite NETs histologically confirmed by biopsy forceps, from August 2013 to April 2018. Patients with GI NETs of $\leq 10 \mathrm{~mm}$ localized to the SM without regional lymph node involvement and/or distant metastasis were included. Patients with indeterminate SELs for which a malignant potential could not be ruled out, including symptomatic SELs, were also enrolled. The inclusion criteria were based upon the recommendations of the European Neuroendocrine Tumor Society [15] and a Japanese nationwide survey [16], which indicated that the risk of metastasis is very low when tumors are localized to the SM layer and are $\leq 10 \mathrm{~mm}$ in size without atypical features or lympho-vascular invasion [6]. The exclusion criteria were an age of $<20$ years, obvious preoperative diagnosis of neuroendocrine carcinoma, and lack of patient consent. All regions were evaluated by routine endosonography (EUS) using a high-frequency $20-\mathrm{MHz}$ miniprobe (UM-3R; Olympus, Tokyo, Japan) and enhanced computed tomography. All data were extracted and compiled into a central database at Kagawa University. The present study was approved by the Clinical Ethics Committee of Kagawa University Hospital (No. H25-030) and each institution in accordance with the Declaration of Helsinki. The study was registered as University Hospital Medical Information Network (UMIN) Clinical Trials Registry Number 000011690 and followed the CONSORT checklist. All patients provided written informed consent to undergo the procedures and participate in the study.

\section{ESD procedure}

All patients were generally placed under conscious or deep sedation with intravenous midazolam $(0.05 \mathrm{mg} / \mathrm{kg})$. Endoscopic submucosal dissection was performed using a single-channel scope with a water jet function (GIF-H260Z or Q260J for upper GI or PCF-Q260AI for rectum; Olympus) and an electrosurgical unit (VIO300D; Erbe Elektromedizin, Tübingen, Germany). A longer transparent cap (Elastic Touch F-030; Top Corp., Tokyo, Japan) or short ST hood (DH29CR; Fujifilm, Tokyo, Japan) was mounted on the tip of the endoscope to allow easy entry into the SM space and a clear view. In the ESD technique for NETs, all operators agreed that the deep SM layer directly above the MP should be dissected. The remaining procedure and applied devices for ESD were chosen at the operator's discretion. First, after marking a circumferential safety margin, a submucosal entry was created by a mucosal incision using submucosal injection of $0.4 \%$ hyaluronate sodium solution (MucoUp; Johnson \& Johnson K.K., Tokyo, Japan) with a needle knife. Submucosal dissection followed by whole circumferential mucosal incision was performed. The tumor was resected by proceeding submucosal dissection. During the procedure, hemostatic forceps were used to manage bleeding and to coagulate exposed vessels in the dissected area. All ESD procedures were performed by five expert endoscopists (H.K., H.M., Y.M., Y.I., and T.Y.) who had successfully performed more than 100 gastric, 10 duodenal, and 50 colorectal ESD procedures.

\section{Outcome measures}

\section{Technical and clinical variables}

The overall en bloc resection rate, curative resection rate, complication rate, and procedure time of all 57 patients were evaluated. These outcomes were analyzed by dividing the included SELs into definite NETs and indeterminate (including symptomatic) SELs. The outcomes were then analyzed on an organ-specific basis (stomach, duodenum, and rectum). En bloc resection was defined as resection of a one-piece specimen, including the marking dots. Curative resection was defined as histological confirmation of the tumor as NET G1 with negative lateral and/or vertical margins and no lymphatic or venous invasion. The procedure time was measured from the submucosal injection to the completion of resection. Complications included the occurrence of any bleeding requiring an endoscopic hemostatic procedure. Perforation was defined as GI wall penetration observed during or after the procedure. A severe complication was defined as the requirement of immediate surgical repair.

\section{Surveillance after ESD}

The mean follow-up period was calculated. The rates and details of salvage operations and recurrence during follow-up were evaluated. All patients were scheduled to undergo over a 5 -year period, regular surveys involving standard endoscopy and computed tomography (chest, abdomen, and pelvis) at 3, 6, and 12 months after ESD and once a year thereafter. Additional surgery was recommended if curative resection had not been achieved as shown by histological examination, if local recurrence, or localized lymph node metastasis were present. Standard chemotherapy was recommended for patients with distant metastasis.

\section{Histological variables}

The overall rates of histologically margin-free resection and the details of histological findings (including the lateral and/ or vertical margin status and lymphatic and vascular invasion) 
were evaluated. These outcomes were also analyzed for each organ (stomach, duodenum, and colorectum). Histologically margin-free resection was defined as negative lateral and vertical margins. Gastrointestinal NETs were histologically categorized into the well-differentiated type, which included NET G1 and NET G2, and the poorly differentiated type (neuroendocrine carcinoma) based on the Ki67 index and mitotic counts. They were also classified based on the World Health Organization TNM classification of well-differentiated endocrine tumor/carcinoma for further staging and treatment [17].

\section{Statistical analysis}

Normally distributed data are presented as median (range). The rates of all outcomes were calculated. These rates were compared between the NETs and indeterminate SELs groups using the two-sided Fisher's exact test. The mean procedure time was compared using the Mann-Whitney U test. A p value of $<0.05$ was considered statistically significant. All statistical analyses were conducted using JMP version 9.0 (SAS Institute Inc., Cary, NC, USA).

\section{RESULTS}

The patients' detailed clinical data are summarized in Table I.

Table I. Demographics and characteristics of patients who underwent ESD for SELs

\begin{tabular}{lccc}
\hline Characteristics & Total & NETs & $\begin{array}{c}\text { Indeterminate } \\
\text { SELs }\end{array}$ \\
\hline Total patients & 57 & 42 & 15 \\
Sex, male/female & $35 / 22$ & $27 / 15$ & $8 / 7$ \\
Age, years & $63(32-86)$ & 63.5 & 57 \\
Location & & & \\
Esophagus & 8 & 0 & 8 \\
Stomach & 7 & 5 & 2 \\
Duodenum & 3 & 3 & 0 \\
Colon & 2 & 0 & 2 \\
Rectum & 37 & 32 & 3 \\
Maximum lesion size on EUS, & $7(2-52)$ & 6 & 11.5 \\
mm & & & \\
\hline Dam & & & \\
\hline
\end{tabular}

Data are presented as n or median (range). ESD: endoscopic submucosal dissection; SEL: subepithelial lesion; NET: neuroendocrine tumor; EUS: endosonography.

In total, 57 consecutive patients ( 35 men, 22 women; median age: 63 years; age range: $32-86$ years) with GI SELs were divided into those with definite NETs $(n=42)$ and indeterminate SELs $(\mathrm{n}=15)$, including 1 symptomatic SEL. Of the 57 SELs, 8 were located in the esophagus, 7 in the stomach, 3 in the duodenum, 2 in the colon, and 37 in the rectum. The median maximum lesion size as measured by EUS was $7 \mathrm{~mm}$ (range: $2-52 \mathrm{~mm}$ ).

The technical and clinical outcomes are summarized in Table II. A flow diagram of the patients' outcomes is shown in Fig. 1.
The rates of en bloc resection and curative resection were $98.2 \%$ and $66.7 \%$ for the overall population $(n=57), 100 \%$ and $61.9 \%$ for patients with NETs $(n=42)$, and $93.3 \%$ and $80.0 \%$ for patients with indeterminate SELs $(n=15)$, respectively. The complication rate in the overall population was $7.7 \%(n=4$; 2 delayed bleedings and 2 micro-perforations); in patients with NETs was $2.4 \%(\mathrm{n}=1$, perforation with $1 \mathrm{~mm}$ in size in a gastric NET which was $10 \mathrm{~mm}$ in maximum lesion size), and in patients with indeterminate SELs was $20 \%$ ( $n=3 ; 2$ bleedings and 1 perforation with $1 \mathrm{~mm}$ in size in a colonic inflammatory granuloma which was $30 \mathrm{~mm}$ in maximum lesion size). All of these complications were managed with endoscopic hemostasis or closure using hemoclips. The median procedure time was 43 $(14-142) \mathrm{min}$ in the overall population $(\mathrm{n}=57), 41.5(17-142)$ min in patients with NETs, and 45 (14-140) min in patients with indeterminate SELs. A salvage operation was performed for five patients $(8.5 \%)$ among the overall population with SELs, five patients (11.9\%) with NETs, and no patients with indeterminate SELs. No patients developed tumor recurrence during the follow-up period (median, 24.5 months; range, 1-60 months).

The organ-specific technical and clinical results of ESD for NETs are shown in Table III. The curative resection rate was poorer in the stomach $(20 \%, \mathrm{n}=5)$ and duodenum $(33 \%, \mathrm{n}=3)$ than in the rectum $(71 \%, \mathrm{n}=34)$. No significant differences were found among the stomach, duodenum, and rectum in terms of complications [20\% (1/5, 1 microperforation) vs. $0 \%$ vs. $0 \%$, respectively] or the median procedure time ( 47 vs. 43 vs. $40 \mathrm{~min}$, respectively) ( $\mathrm{p}>0.05)$. Salvage operations were performed in the stomach for $40 \%(2 / 5)$ of patients, in the duodenum for $33.3 \%(1 / 3)$, and in the rectum for $5.9 \%(2 / 34)$. A severe complication involving anastomotic dehiscence that progressed to sepsis occurred in one patient who underwent a salvage operation. Eleven of 16 patients without curative resection did not undergo a salvage operation because of refusal to undergo colostomy $(n=7)$, economic issues $(n=2)$, and severe comorbidities $(n=2)$.

The histological results of ESD for NETs in the overall population and for each organ are shown in Table IV. NETs were histologically categorized into NET G1 $(n=36,86 \%)$ and NET G2 ( $n=6,14 \%)$. ESD achieved a high negative vertical margin rate $(93 \%, n=39)$, and the positive lateral margin, lymphatic invasion, and venous invasion rates were $5 \%$ $(n=2), 5 \%(n=2)$, and $17 \%(n=7)$, respectively. With respect to organ-specific outcomes, the rate of histologically margin-free resection was the lowest in the duodenum (67\%). A positive lateral margin was present in two patients $(6 \%)$ with rectal tumors. The minimum surgical margin, measured from the en bloc specimens (defined as the shortest distance between the edge of resected specimen and the edge of tumor elevation) in these two patients with positive lateral margins was shorter than that in patients who had rectal tumors with negative lateral margins (mean, 4.5 vs. $12.6 \mathrm{~mm}$, respectively). A representative case of ESD for a rectal NET is shown in Fig. 2.

Detailed results of ESD for indeterminate SELs $(n=15)$ are shown in Table V. Histological examination of these patients' specimens finally confirmed granular cell tumors ( $n=7 ; 6$ esophagus, 1 stomach), leiomyoma ( $n=1$, esophagus), hemangioma ( $\mathrm{n}=1$, esophagus), symptomatic lipoma with 
Table II. Technical and clinical results of ESD for SELs

\begin{tabular}{lcccc}
\hline Variables & $\begin{array}{c}\text { Overall } \\
(\mathrm{n}=57)\end{array}$ & $\begin{array}{c}\text { NETs } \\
(\mathrm{n}=42)\end{array}$ & $\begin{array}{c}\text { Indeterminate } \\
\text { SELs }(\mathrm{n}=15)\end{array}$ & $\mathrm{p}$ \\
\hline En bloc resection & $56(98.2)$ & $42(100)$ & $14(93.3)$ & $0.2632^{*}$ \\
Curative resection & $38(66.7)$ & $26(61.9)$ & $12(80.0)$ & $0.3388^{*}$ \\
Complications & & & & \\
$\quad 4(7.7)$ & $1(2.4)$ & $3(20.0)$ & $0.0518^{*}$ \\
$\quad$ All & $2(3.5)$ & $1(2.4)$ & $1(6.7)$ & $0.4605^{*}$ \\
$\quad$ Perforation & $2(3.5)$ & 0 & $2(13.0)$ & $0.0658^{*}$ \\
$\quad$ Bleeding & 0 & 0 & 0 & \\
$\quad$ Severe & $43(14-142)$ & 41.5 & 45 & $0.9133^{*}$ \\
Procedure time, min & $5(8.8)$ & $5(11.9)$ & 0 & $0.3111^{*}$ \\
Salvage operation & 0 & 0 & 0 & \\
Local recurrences of ESD scar & 0 & 0 & 0 & \\
Local lymph node metastasis & 0 & 0 & 0 & \\
Recurrence during follow-up & $24.5(1-60)$ & 25.5 & 16 & \\
Follow-up period, months & &
\end{tabular}

Data are presented as $\mathrm{n}(\%)$ or median (range). ESD: endoscopic submucosal dissection; SEL: subepithelial lesion; NET: neuroendocrine tumor. ${ }^{\sharp}$ Fisher's exact test; ${ }^{*}$ Mann-Whitney U test.

ball valve syndrome, defined as the condition that the tumor causes gastric outlet obstruction ( $\mathrm{n}=1$, stomach), inflammatory granuloma ( $\mathrm{n}=1$, ascending colon), phlebolith ( $\mathrm{n}=1$, sigmoid), schwannoma ( $\mathrm{n}=1$, rectum), lymphoid hyperplasia $(\mathrm{n}=1$, rectum), and MALT lymphoma ( $\mathrm{n}=1$, rectum).

Although curative resection could not be achieved in 2 of 15 patients (13.3\%), none of these 15 patients developed recurrent lesions during the follow-up period (median, 16 months). A representative case of ESD for a rectal indeterminate SEL is shown in Fig. 3.

\section{DISCUSSION}

This is the first prospective multicenter study to investigate the outcomes of ESD for SELs located within the SM layer with organ-specific results of NETs. In the present study, we identified four main important clinical findings. First, in contrast to previous reports, ESD for small NETs of $\leq 10 \mathrm{~mm}$ does not provide a high rate of curative resection according to the histological results of risk factors for metastasis. Gastric and duodenal ESD for NETs may be especially limited in terms of achieving curative resection. Second, a sufficient lateral margin is necessary when performing ESD. Third, ESD followed by surveillance demonstrates acceptable mid-term outcomes for non-curative NETs. Fourth, the clinician must pay attention to the risk of ESD complications in patients with indeterminate SELs.

This study was designed based on the concept that ESD can be a reasonable option for complete resection of SELs located within the SM layer. Endoscopic submucosal dissection

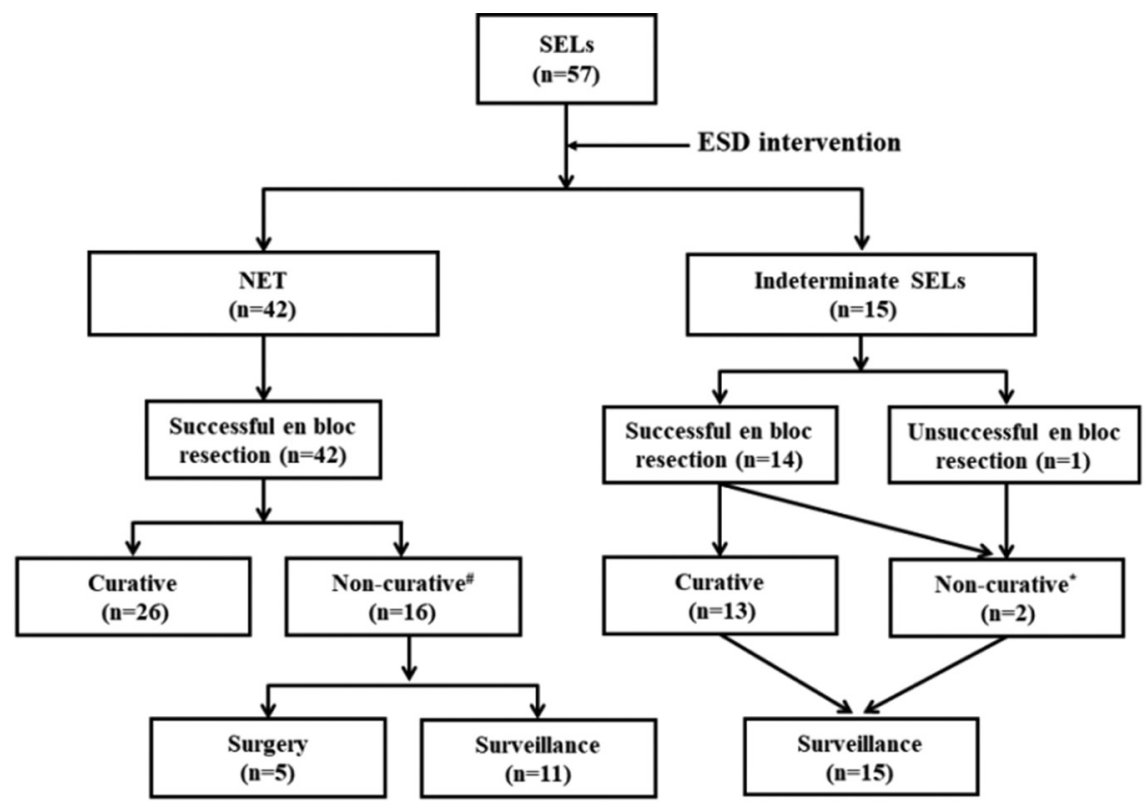

Fig. 1. Flow diagram of patient outcomes. "Non-curative factors (and/or): NET G2 $(n=6)$, positive lateral margin $(n=2)$, positive vertical margin $(n=2)$, positive lymphatic invasion $(n=2)$, positive venous invasion $(n=7) .{ }^{*}$ Unevaluable lateral and vertical margins $(n=2)$. 
Table III. Organ-specific technical and clinical results of ESD for NETs

\begin{tabular}{|c|c|c|c|c|}
\hline Variables & Overall $(n=42)$ & $\operatorname{Stomach}(n=5)$ & Duodenum $(n=3)$ & $\operatorname{Rectum}(\mathrm{n}=34)$ \\
\hline En bloc resection & $42(100)$ & $5(100)$ & $3(100)$ & $34(100)$ \\
\hline Curative resection & $26(62)$ & $1(20)$ & $1(33)$ & $24(71)$ \\
\hline \multicolumn{5}{|l|}{ Complications } \\
\hline Perforation & $1(2.4)$ & $1(20)$ & 0 & 0 \\
\hline Bleeding & 0 & 0 & 0 & 0 \\
\hline Procedure time, $\min$ & $43(14-142)$ & $47(10-120)$ & $43(30-142)$ & $40(17-125)$ \\
\hline Salvage operation & $5(8.8)$ & $2(40)$ & $1(33)$ & $2(5.9)$ \\
\hline Local recurrences & 0 & 0 & 0 & 0 \\
\hline $\begin{array}{l}\text { Local lymph node } \\
\text { metastasis }\end{array}$ & 0 & 0 & 0 & 0 \\
\hline Complications & 0 & 0 & 0 & $1(2.9)^{\#}$ \\
\hline
\end{tabular}

is performed to achieve curative resection of small NETs or diagnostic resection of indeterminate SELs. We herein discuss ESD for NETs and indeterminate SELs separately.

\section{NETs}

A prospective comparative study revealed that ESD was superior to EMR, showing that the rate of histologically complete resection of rectal NETs was $90 \%$ for ESD versus $71 \%$ for EMR [18]. The rate of histologically complete resection by ESD was higher than previous reported rates of $38 \%$ and $86 \%$ rates using modified EMR methods (EMR with a ligation device [19] and cap-assisted EMR [20]). The main reason for this difference is that ESD enables deep submucosal dissection directly above the MP because NETs invade into the SM layer. A multivariate analysis of 345 nationwide cases showed that NETs of $\leq 10 \mathrm{~mm}$ without lymphatic invasion could be curatively treated by endoscopic resection [16]. Moreover, the analysis revealed that a tumor size of $>10 \mathrm{~mm}$ and positive lymphatic invasion were independently predictive of lymph node metastasis. However, the proportion of $\leq 10 \mathrm{~mm}$ NETs with venous invasion and the proportion of these tumors that develop metastasis remain unclear. Our data revealed the rate of each non-curative factor in overall NETs: NET G2 (14\%), positive lateral margin (5\%), positive vertical margin (5\%), positive lymphatic invasion (5\%), and positive venous invasion (17\%), demonstrating a curative resection rate of only $62 \%$. As shown in Table IV, gastric NETs had the highest rates of NET G2 $(40 \%, 2 / 5)$ and positive venous invasion $(60 \%, 3 / 5)$, suggesting that gastric NETs might have a high malignant potential even when small $(\leq 10 \mathrm{~mm})$. However, the 5-year survival rate of patients with gastric NET G1 of $\leq 10 \mathrm{~mm}$ with neither infiltration of the MP nor lympho-vascular invasion

Table IV. Histopathological results of ESD for NETs

\begin{tabular}{lcccc}
\hline Variables & Overall $(\mathrm{n}=42)$ & Stomach $(\mathrm{n}=5)$ & Duodenum $(\mathrm{n}=3)$ & Rectum $(\mathrm{n}=34)$ \\
\hline NET & & & & \\
Grade 1 & $36(86)$ & $3(60)$ & $3(100)$ & $30(88)$ \\
$\quad$ Grade 2 & $6(14)$ & $2(40)$ & $0(0)$ & $4(12)$ \\
Histologically margin-free & $37(88)$ & $5(100)$ & $2(67)$ & $30(88)$ \\
Lateral margin & & & & \\
$\quad$ Positive & $2(5)$ & 0 & 0 & $2(6)$ \\
$\quad$ Negative & $39(93)$ & $5(100)$ & $2(67)$ & $32(94)$ \\
$\quad$ Unevaluable & $1(2)$ & 0 & $1(33)$ & 0 \\
Vertical margin & & & & $1(3)$ \\
$\quad$ Positive & $2(5)$ & 0 & $1(33)$ & $32(94)$ \\
$\quad$ Negative & $39(93)$ & $5(100)$ & $2(67)$ & $1(3)$ \\
$\quad$ Unevaluable & $1(2)$ & 0 & 0 & $1(3)$ \\
Lymphatic invasion & & & & $33(97)$ \\
$\quad$ Positive & $2(5)$ & 0 & $2(67)$ & $3(9)$ \\
$\quad$ Negative & $40(95)$ & $5(100)$ & & $31(91)$ \\
Venous invasion & & & $2(67)$ & \\
$\quad$ Positive & $7(17)$ & $3(60)$ & $2(40)$ & \\
Negative & $35(83)$ & & & \\
\hline
\end{tabular}

Data are presented as n (\%). ESD: endoscopic submucosal dissection; NET: neuroendocrine tumor. 

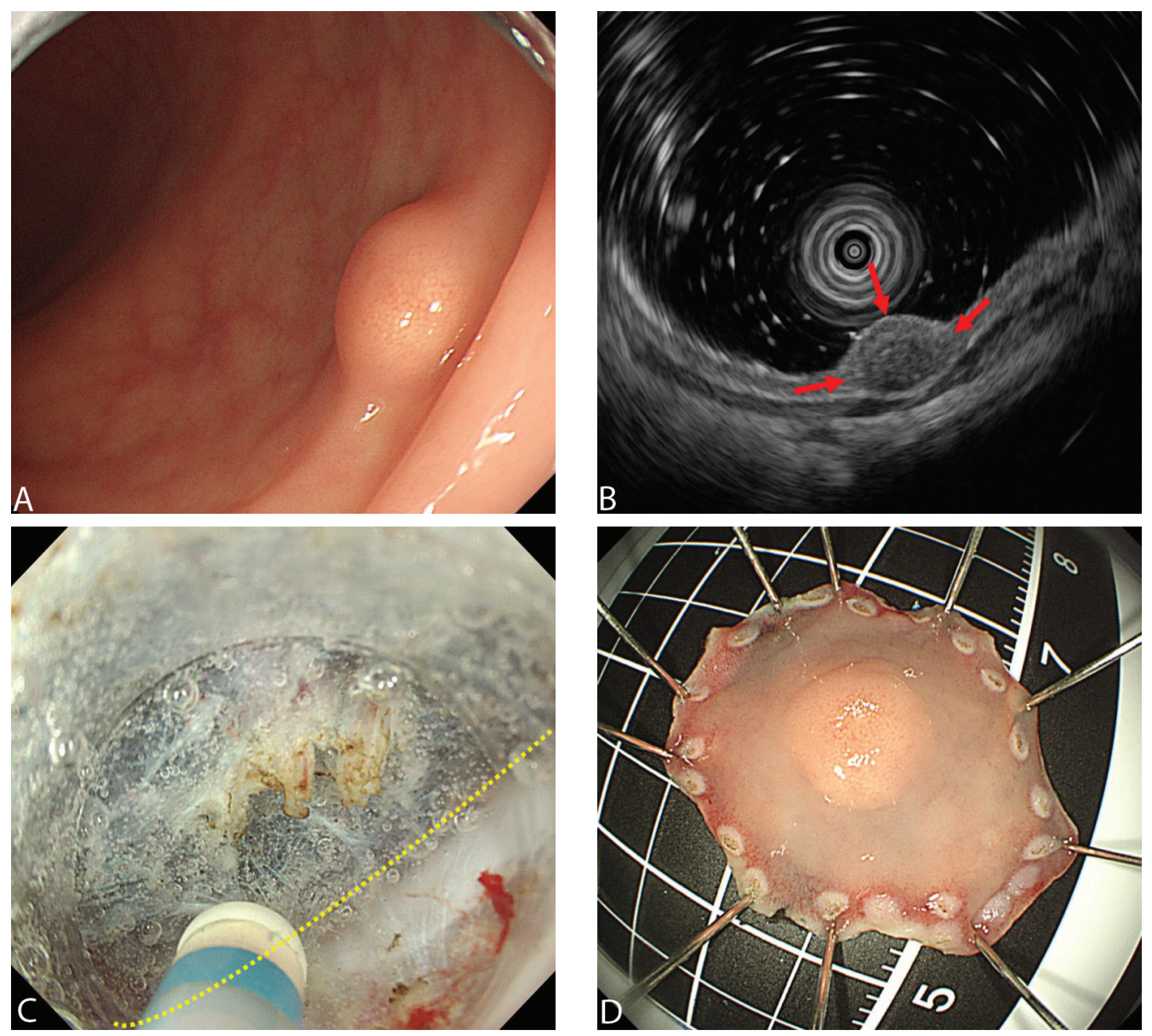

Fig. 2. Representative case of endoscopic submucosal dissection (ESD) for a rectal neuroendocrine tumor (NET). (A) Endoscopic image showing a small NET located in the lower rectum. (B) Endosonography revealing a hypoechoic mass located within the submucosal layer (red arrows) (maximum diameter, $6.7 \mathrm{~mm}$ ). (C) Endoscopic image showing the submucosal dissection directly above the muscle layer (yellow line) to maintain a negative vertical margin. (D) Macroscopic image of the en bloc specimen $(22 \times 20 \mathrm{~mm})$ obtained by ESD, resulting in the curative resection of well-differentiated NET.

reaches $98 \%$, indicating a low risk of tumor-related death [21]. Accordingly, endoscopic resection might be acceptable as a final treatment option in some patients with small gastric NET G2 without other risk factors for metastatic disease. According to a recent literature analysis of rectal NETs, salvage surgery may be better indicated based on the finding that the development of metastases might be associated with a mitotic index of $\geq 2$ mitotic figures per 10 high-power fields than with the Ki-67 proliferation index [22]. In the present study, a moderate rate of histologically margin-free resection in the duodenum was achieved (67\%). However, the procedure time in one patient was $142 \mathrm{~min}$ because the lesion was located in the anterior wall of the duodenal bulb directly over the pyloric ring, and maneuvering the scope was too difficult. Endoscopic submucosal dissection is still challenging with respect to the handling of duodenal tumors. This difficulty is associated with technical difficulties because of the anatomical features of the duodenum (thin wall and insufficient submucosal lifting). In addition, ESD increases the risk of duodenal perforation [23]. Therefore, conservative management may be a better option for patients of advanced age who have multiple morbidities and asymptomatic duodenal NET G1 of $\leq 10 \mathrm{~mm}$ [24]. We recommend the performance of duodenal ESD for NETs in referral centers. In the future, duodenal endoscopic fullthickness resection using an over-the-scope clip system is expected to emerge as a newly advanced and reliable procedure to overcome the technical aspects of the operation and the difficulties associated with NETs features [25, 26]. Although the en bloc resection rate of rectal NETs was $100 \%$, the curative resection rate was lower $(71 \%)$ than previous reported $(90 \%)$ [18] and was similar to that of various EMR procedures (72-74\%) [27]. The curative resection rate seems to be related to non-curative factors regardless of endoscopic skills. Our data showed a positive lateral margin in two patients $(6 \%)$ with rectal tumors. The minimum surgical margin, measured from the en bloc specimens (defined as the shortest distance between the edge of resected specimen and the edge of tumor elevation) in these two patients with positive lateral margins was shorter than that in patients who had rectal tumors with negative lateral margins (mean, $4.5 \mathrm{vs.} 12.6 \mathrm{~mm}$, respectively). Because NETs inflate into the SM and might unexpectedly grow as a dumbbell-type lesion, a sufficient lateral margin of $\geq 5 \mathrm{~mm}$ is recommended. In the rectal ESD of the present study, the relatively larger safety margin may have prolonged the procedure time (mean, $40 \mathrm{~min}$ ) compared with that in another report (mean, $11.4 \mathrm{~min}$ ) [18]. The longer procedure 
Table V. Detailed results of ESD for indeterminate SELs $(\mathrm{n}=15)$

\begin{tabular}{|c|c|c|c|c|c|c|}
\hline Case & Organ/location & $\begin{array}{c}\text { Maximum } \\
\text { lesion size } \\
(\mathrm{mm})\end{array}$ & $\begin{array}{l}\text { En bloc } \\
\text { resection }\end{array}$ & Curative resection & Complications & Final diagnosis \\
\hline 1 & Esophagus/Ce & 6 & Yes & Yes & No & Granular cell tumor \\
\hline 2 & Esophagus/Te & 4.5 & Yes & Yes & No & Granular cell tumor \\
\hline 3 & Esophagus/Te & 7.2 & Yes & Yes & No & Granular cell tumor \\
\hline 4 & Esophagus/Te & 4 & Yes & Yes & No & Granular cell tumor \\
\hline 5 & Esophagus/Te & 9 & Yes & Yes & No & Granular cell tumor \\
\hline 6 & Esophagus/Te & 7 & Yes & Yes & Delayed bleeding & Granular cell tumor \\
\hline 7 & Esophagus/Ae & 20 & Yes & Yes & No & Leiomyoma \\
\hline 8 & Esophagus/Ae & 15 & Yes & Yes & No & Hemangioma \\
\hline 9 & Stomach/body & 10 & Yes & Yes & No & Granular cell tumor \\
\hline 10 & Stomach/antrum & 28 & Yes & Yes & Delayed bleeding $\$$ & Symptomatic lipoma \\
\hline 11 & Colon/ascending & 30 & No & No (HMX, VMX) & Microperforation\& & Inflammatory granuloma \\
\hline 12 & Colon/sigmoid & 7 & Yes & Yes & No & Phlebolith \\
\hline 13 & Rectum/Ra & 10 & Yes & Yes & No & Schwannoma \\
\hline 14 & Rectum/Rb & 22 & Yes & Yes & No & Lymphoid hyperplasia \\
\hline 15 & Rectum/Rb & 52 & Yes & No (HMX, VMX) & No & MALT lymphoma \\
\hline
\end{tabular}

ESD: endoscopic submucosal dissection; SEL: subepithelial lesion; Ce: cervical esophagus; Te: thoracic esophagus; Ae: abdominal esophagus; Ra: upper rectum; Rb: lower rectum; VMX: unevaluable vertical margin; HMX: unevaluable horizontal margin; MALT: mucosa-associated lymphoid tissue; \$Uptake of anticoagulant; \&1 mm in the perforation size.

time may have also been influenced by the sufficient time spent to achieve safe and reliable deep submucosal dissection.

With respect to ESD followed by surveillance, 11 of 16 patients who did not achieve curative resection did not undergo a salvage operation for unavoidable reasons. NETs G1 of $\leq 10$ $\mathrm{mm}$ are reportedly associated with metastases in $2 \%$ of cases and have a 5 -year overall survival rate of $95 \%$ to $100 \%$ [28]. In the present study, no recurrences occurred in all patients during the 26-month median follow-up period. Although patients with tumors of $\leq 10 \mathrm{~mm}$ and non-curative factors after ESD should undergo continuous long-term surveillance, our study demonstrated that the mid-term outcomes were acceptable for non-curative NETs. Non-curative factors related to metastasis in NETs of $\leq 10 \mathrm{~mm}$ require further investigation.

\section{Indeterminate SELs}

Some SELs located within the SM layer have malignant potential. When tissue sampling errors occur, endoscopic resection, which is performed for both diagnosis and treatment, can be a better alternative. Several studies have shown the efficacy of submucosal tunnel endoscopic resection [29] over ESD [30] for resection of SELs originating from the MP layer. For those located in the MP layer, according to a literature review, the success rate of en bloc resection was higher with submucosal tunnel endoscopic resection $(83.3 \%-100 \%)$ than with ESD (64\%-75\%), and the complication rates were similar (0-16.7\% and $0-14.5 \%$, respectively) [31]. Thus, ESD is a limited option for resecting SELs located within the MP layer. No studies and few case reports have described ESD for symptomatic indeterminate SELs located within the SM layer [11-14]. In the present study, all cases were finally diagnosed by histological examination of ESD specimens, demonstrating the efficacy of diagnostic ESD. The rates of en bloc resection, curative resection, and complications were $93.3 \%, 80.0 \%$, and $20 \%$, respectively, for indeterminate SELs. Two patients with colorectal lesions (one with an inflammatory granuloma and one with MALT lymphoma) did not achieve margin-negative resection because of severe fibrosis and poor visibility of the SM. A microperforation with $1 \mathrm{~mm}$ in size occurred in the patient with the inflammatory granuloma, which was $30 \mathrm{~mm}$ in maximum lesion size and located in the ascending colon. Thus, colonic ESD, which necessitates advanced skills, may be limited in some cases of indeterminate SELs. However, ESD showed a higher curative resection rate for indeterminate SELs than for NETs ( $80 \%$ vs. $61.9 \%$, respectively). This result is probably associated with the inclusion of almost benign tumors requiring no detailed histological evaluations of lymphovascular invasion. Among the SELs in this study, curative resection was achieved for all seven granular cell tumors (six in the esophagus, one in the stomach). The median size was small $(6.5 \mathrm{~mm})$ and the median procedure time was short (28.5 min). Thus, ESD can become a curative option if a granular cell tumor is suspected. Furthermore, ESD was effective for curative resection of symptomatic gastric lipoma. Although the complication rate of $20 \%$ was higher than that for NETs $(2.4 \%)$, all cases were endoscopically managed. Consequently, ESD can be both a diagnostic and therapeutic option for indeterminate SELs located within the SM.

This study had three main limitations. First, the number of patients with indeterminate SELs was small. Second, although mid-term surveillance was performed, 5-year surveillance is more desirable for NETs. Third, a comparison arm is lacking in this study. Thus, a multi-center prospective study with a large number of participants must be conducted to emphasize the efficacy and safety of ESD by comparing the two groups between ESD and EMR. 

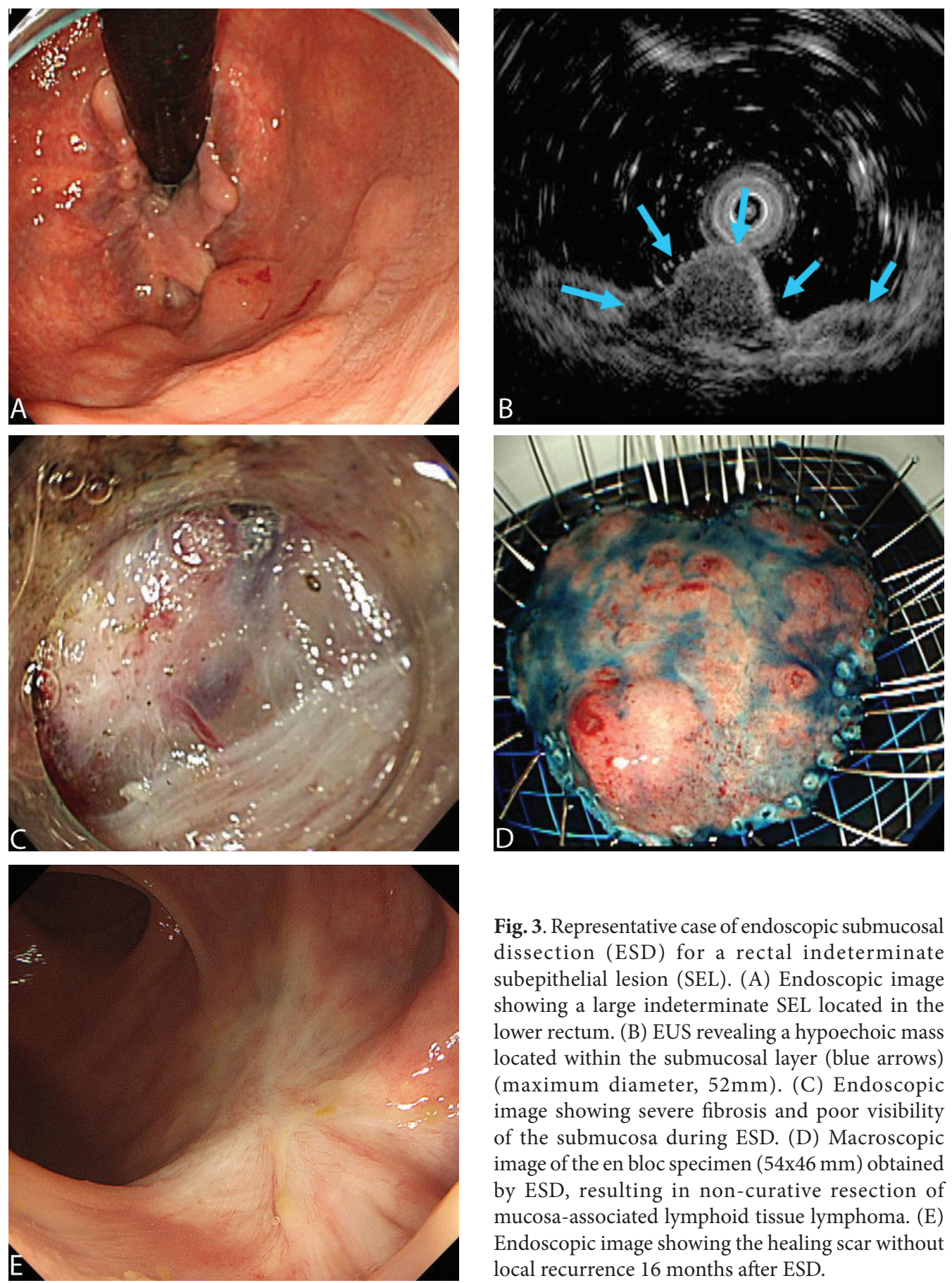

Fig. 3. Representative case of endoscopic submucosal dissection (ESD) for a rectal indeterminate subepithelial lesion (SEL). (A) Endoscopic image showing a large indeterminate SEL located in the lower rectum. (B) EUS revealing a hypoechoic mass located within the submucosal layer (blue arrows) (maximum diameter, $52 \mathrm{~mm}$ ). (C) Endoscopic image showing severe fibrosis and poor visibility of the submucosa during ESD. (D) Macroscopic image of the en bloc specimen (54x46 mm) obtained by ESD, resulting in non-curative resection of mucosa-associated lymphoid tissue lymphoma. (E) Endoscopic image showing the healing scar without local recurrence 16 months after ESD.

\section{CONCLUSION}

Endoscopic submucosal dissection for SELs located within the SM layer can be a feasible diagnostic and therapeutic option. ESD followed by conservative treatment may be a reasonable strategy for NET G1 of $\leq 10 \mathrm{~mm}$, even when non-curative factors are present. However, gastric and duodenal ESD for NETs may be limited in terms of its curative and technical aspects.

Conflicts of interest: None to declare.

Author' contributions: H.K., Y.M.: study concept and design; T.Yamada, N.N., N.K., T.C., T.Yachida, H.M.: data acquisition;

Y.I., M.T.: data interpretation; S.F.: statistical analysis; K.O., Y.S. supplied the material; K.F., J.T. drafted the manuscript; T.M.: study supervision.

Acknowledgements: We thank Edanz Group (www.edanzediting. com/ac) for editing a draft of this manuscript. This work was supported by the Japan Society for the Promotion of Science (JSPS) KAKENHI Grant Number 15K06855 and by the Alumni Association of Faculty of Medicine, Kagawa University, Grant Number 27-1. These people who funded this study had no role in the study design, data collection or analysis, decision to publish, or the preparation of the manuscript. 


\section{REFERENCES}

1. Uedo N, Takeuchi Y, Ishihara R. Endoscopic management of early gastric cancer: endoscopic mucosal resection or endoscopic submucosal dissection: data from a Japanese high-volume center and literature review. Ann Gastroenterol 2012;25:281-290.

2. Ono H, Yao K, Fujishiro M, et al. Guidelines for endoscopic submucosal dissection and endoscopic mucosal resection for early gastric cancer. Dig Endosc 2016;28:3-15. doi:10.1111/den.12518

3. Toyonaga T, Man-I M, Morita Y, Azuma T. Endoscopic submucosal dissection (ESD) versus simplified/hybrid ESD. Gastrointest Endosc Clin N Am 2014;24:191-199. doi:10.1016/j.giec.2013.11.004

4. Plöckinger U, Rindi G, Arnold R, et al. Guidelines for the diagnosis and treatment of neuroendocrine gastrointestinal tumours. A consensus statement on behalf of the European Neuroendocrine Tumour Society (ENETS). Neuroendocrinology 2004;80:394-424 doi:10.1159/000085237

5. Modlin IM, Oberg K, Chung DC, et al. Gastroenteropancreatic neuroendocrine tumours. Lancet Oncol 2008;9:61-72. doi:10.1016/ S1470-2045(07)70410-2

6. Park HW, Byeon JS, Park YS, et al. Endoscopic submucosal dissection for treatment of rectal carcinoid tumors. Gastrointest Endosc 2010;72:143149. doi:10.1016/j.gie.2010.01.040

7. Kobara H, Mori H, Chei L, Fujihara S, Nishiyama N, Masaki T. The advantage of an endoscopic submucosal tunneling technique for rectal carcinoid tumors. Gut Liver 2017;11:735-737. doi:10.5009/gnl16580

8. Matsumoto T, Iida M, Suekane H, Tominaga M, Yao T, Fujishima M. Endoscopic ultrasonography in rectal carcinoid tumors: contribution to selection of therapy. Gastrointest Endosc 1991;37:539-542. doi:10.1016/ s0016-5107(91)70824-9

9. Zhou PH, Yao LQ, Qin XY, et al. Advantages of endoscopic submucosal dissection with needle-knife over endoscopic mucosal resection for small rectal carcinoid tumors: a retrospective study. Surg Endosc 2010;24:2607-2612. doi:10.1007/s00464-010-1016-Z

10. Zhong DD, Shao LM, Cai JT. Endoscopic mucosal resection vs endoscopic submucosal dissection for rectal carcinoid tumours: a systematic review and meta-analysis. Colorectal Dis 2013;15:283-291. doi:10.1111/codi.12069

11. Kojima T, Takahashi H, Parra-Blanco A, Kohsen K, Fujita R. Diagnosis of submucosal tumor of the upper GI tract by endoscopic resection. Gastrointest Endosc 1999;50:516-522. doi:10.1016/s00165107(99)70075-1

12. Waxman I, Saitoh Y, Raju GS, et al. High-frequency probe EUS-assisted endoscopic mucosal resection: a therapeutic strategy for submucosal tumors of the GI tract. Gastrointest Endosc 2002;55:44-49. doi:10.1067/ mge.2002.119871

13. Yoshida T, Fujisaki J, Suganuma T, et al. Successful en bloc resection of a $5 \mathrm{~cm}$ symptomatic sessile gastric lipoma by endoscopic submucosal dissection. Dig Endosc 2012;24:282. doi:10.1111/j.14431661.2011.01199.x

14. Kobara H, Mori H, Masaki T. Successful en bloc resection of an esophageal hemangioma by endoscopic submucosal dissection. Endoscopy 2012;44:E134-E135. doi:10.1055/s-0030-1256703

15. Delle Fave G, O’Toole D, Sundin A, et al; Vienna consensus conference participants. ENETS consensus guidelines update for gastroduodenal neuroendocrine neoplasms. Neuroendocrinology 2016;103:119-124. doi: $10.1159 / 000443168$

16. Konishi T, Watanabe T, Kishimoto J, et al; Japanese Society for Cancer of the Colon and Rectum. Prognosis and risk factors of metastasis in colorectal carcinoids: results of a nationwide registry over 15 years. Gut 2007;56:863-868. doi:10.1136/gut.2006.109157

17. Rindi G, Klimstra DS, Abedi-Ardekani B, et al. A common classification framework for neuroendocrine neoplasms: an International Agency for Research on Cancer (IARC) and World Health Organization (WHO) expert consensus proposal. Mod Pathol 2018;31:1770-1786. doi:10.1038/s41379-018-0110-y

18. Park HW, Byeon JS, Park YS, et al. Endoscopic submucosal dissection for treatment of rectal carcinoid tumors. Gastrointest Endosc 2010;72:143149. doi:10.1016/j.gie.2010.01.040

19. Ono A, Fujii T, Saito Y, et al. Endoscopic submucosal resection of rectal carcinoid tumors with a ligation device. Gastrointest Endosc 2003;57:583-587. doi:10.1067/mge.2003.142

20. Nagai T, Torishima R, Nakashima H, et al. Saline-assisted endoscopic resection of rectal carcinoids: cap aspiration method versus simple snare resection. Endoscopy 2004;36:202-205. doi:10.1055/s-2004-814248

21. Ruszniewski P, Delle Fave G, Cadiot G, et al. Well-differentiated gastric tumors/carcinomas. Neuroendocrinology 2006;84:158-164. doi:10.1159/000098007

22. de Mestier L, Brixi H, Gincul R, Ponchon T, Cadiot G. Updating the management of patients with rectal neuroendocrine tumors. Endoscopy 2013;45:1039-1046. doi:10.1055/s-0033-1344794

23. Gincul R, Ponchon T, Napoleon B, et al. Endoscopic treatment of sporadic small duodenal and ampullary neuroendocrine tumors. Endoscopy 2016;48:979-986. doi:10.1055/s-0042-112570

24. Scherübl H, Cadiot G. Early gastroenteropancreatic neuroendocrine tumors: endoscopic therapy and surveillance. Visc Med 2017;33:332338. doi:10.1159/000459404

25. Schmidt A, Meier B, Cahyadi O, Caca K. Duodenal endoscopic fullthickness resection (with video). Gastrointest Endosc 2015;82:728-733. doi:10.1016/j.gie.2015.04.031

26. Kobara H, Mori H, Nishiyama N, et al. Over-the-scope clip system: a review of 1517 cases over 9 years. J Gastroenterol Hepatol 2019;34:2230. doi:10.1111/jgh.14402

27. Kim J, Kim JH, Lee JY, Chun J, Im JP, Kim JS. Clinical outcomes of endoscopic mucosal resection for rectal neuroendocrine tumor. BMC Gastroenterol 2018;18:77. doi:10.1186/s12876-018-0806-y

28. Bertani E, Ravizza D, Milione M, et al. Neuroendocrine neoplasms of rectum: a management update. Cancer Treat Rev 2018;66:45-55. doi:10.1016/j.ctrv.2018.04.003

29. Xu MD, Cai MY, Zhou PH, et al. Submucosal tunneling endoscopic resection: a new technique for treating upper GI submucosal tumors originating from the muscularis propria layer (with videos). Gastrointest Endosc 2012;75:195-199. doi:10.1016/j.gie.2011.08.018

30. Lee IL, Lin PY, Tung SY, Shen CH, Wei KL, Wu CS. Endoscopic submucosal dissection for the treatment of intraluminal gastric subepithelial tumors originating from the musculais propria layer. Endoscopy 2006;38:1024-1028. doi:10.1055/s-2006-944814

31. Kobara H, Mori H, Rafiq K, et al. Submucosal tunneling techniques: current perspectives. Clin Exp Gastroenterol 2014;7:67-74. doi:10.2147/ CEG.S43139 